EPJ manuscript No.

(will be inserted by the editor)

CERN-TH/2002-082

TTP02-03

\title{
The radiative return at small angles: virtual corrections
}

\author{
Johann H. Kühn ${ }^{1,2 a}$ and Germán Rodrigo ${ }^{1 b}$ \\ 1 Theory Division, CERN, CH-1211 Geneva 23, Switzerland. \\ 2 Institut für Theoretische Teilchenphysik, Universität Karlsruhe, D-76128 Karlsruhe, Germany.
}

Received: April 20, 2002

\begin{abstract}
Virtual corrections for electron-positron annihilation into one real and one off-shell photon of invariant mass $Q^{2}$ are evaluated. Special attention is paid to those configurations where the real photon is collinear with the beam direction. This calculation is an important ingredient of a Monte Carlo program, which simulates events with tagged photons from initial-state radiation, including NLO corrections.
\end{abstract}

\section{Introduction}

The total cross section for electron-positron annihilation into hadrons is one of the fundamental observables in particle physics. Its high energy behaviour provides one of the first and still most convincing arguments for the pointlike nature of quarks. Its normalization was evidence for the existence of quarks of three different colours, and the recent, precise measurements even allow for an excellent determination of the strong coupling at very high [1] and intermediate energies (e.g. [2] and refs. therein) through the influence of QCD corrections.

Weighted integrals over the cross section with properly chosen kernels are, furthermore, a decisive input for electroweak precision tests. This applies, for example, to the electromagnetic coupling at higher energies or to the anomalous magnetic moment of the muon.

Of particular importance for these two applications is the low energy region, say from threshold up to centre-ofmass (cms) energies of approximately $3 \mathrm{GeV}$ and $10 \mathrm{GeV}$, respectively. Recent measurements based on energy scans between 2 and $5 \mathrm{GeV}$ have improved the accuracy in part of this range. However, similar, or even further improvements below $2 \mathrm{GeV}$ would be highly welcome. The region between $1.4 \mathrm{GeV}$ and $2 \mathrm{GeV}$, in particular, is poorly studied and no collider will cover this region in the near future. Improvements or even an independent cross-check of the precise measurements of the pion form factor in the low energy region by the CMD2 and DM2 collaborations would be extremely useful, since this dominates in the analysis of the muon anomalous magnetic moment.

\footnotetext{
${ }^{a}$ e-mail: jk@particle.uni-karlsruhe.de

b Supported in part by E.U. TMR grant HPMF-CT-200000989; e-mail: german.rodrigo@cern.ch
}

Experiments at present electron-positron colliders operate mostly at fixed energies, albeit with enormous luminosity, with BaBar and BELLE at $10.6 \mathrm{GeV}$ and KLOE at $1.02 \mathrm{GeV}$ as most prominent examples.

This peculiar feature allows the use of the radiative return, i.e. the reaction

$$
e^{+}\left(p_{1}\right)+e^{-}\left(p_{2}\right) \rightarrow \gamma(k)+\gamma^{*}(Q)(\rightarrow \text { hadrons }),
$$

to explore a wide range of $Q^{2}$ in a single experiment [3,4, 5, 6, $6,8,9,9$.

Nominally masses of the hadronic system between $2 m_{\pi}$ and the cms energy of the experiment are accessible. In practice it is useful to consider only events with a hard photon - tagged or untagged - to clearly identify the reaction, which lowers the energy significantly.

The study of events with photons emitted under both large and small angles, and thus at a significantly enhanced rate, is particularly attractive for the $\pi^{+} \pi^{-}$final state with its clear signature, an investigation performed at present at $\mathrm{DA} \Phi \mathrm{NE}, 10,11,12$. Events with a tagged photon, emitted under a large angle with respect to the beam, have a clear signature and are thus particularly suited to the analysis of hadronic final states of higher multiplicity 13.

The inclusion of radiative corrections is essential for the precise extraction of the cross section, which is necessarily based on a Monte Carlo simulation. A first program, called EVA, was constructed some time ago [3]. It includes initial-state radiation (ISR), final-state radiation (FSR), their interference, and the dominant radiative corrections from additional collinear radiation through structure function techniques [14].

The complete NLO corrections have recently been implemented in a program called PHOKHARA [15]. Both programs, however, were designed to simulate reactions 
with tagged photons, i.e. at least one photon was required to be emitted under large angles.

An important ingredient in the extension of the NLO Monte Carlo program PHOKHARA to small photon angles is the evaluation of the virtual corrections to the reaction (11) in the limit $m_{e}^{2} / s \ll 1$, which are equally valid for large and small angles. Compact results for the oneloop two-, three- and four-point functions that enter this calculation can be found in the literature [16, 17] for arbitrary values of $m_{e}^{2} / s$. However, the combination of these analytical expressions with the relevant coefficients is numerically unstable in the limit of small mass and angles. A compact, numerically stable result, valid for an arbitrarily small photon angle, is therefore required. As a consequence of the highly singular kinematic coefficients, terms proportional to $m_{e}^{2}$ and even $m_{e}^{4}$ must be kept in the expansion, which after angular integration will contribute to the total cross section even in the limit $m_{e}^{2} / s \rightarrow 0$.

The present paper extends the analysis of Ref. 18 where the corrections from virtual and soft photons were presented for the case of large angles. In section 2 we recall the basic definitions and describe the systematic procedure used in the expansion of the results for small $\mathrm{m}_{e}^{2} / \mathrm{s}$ and small angles simultaneously. The analytic results for real and imaginary parts of the leptonic tensor, expressed in an angular momentum basis, are presented in section 3 and compared with results for related quantities that can be found in the literature. After summation over the polarizations of the virtual photons, our result agrees with the one of Berends, Burgers and van Neerven 20,21]. The result of Kuraev, Merenkov and Fadin 222 for the real part of the tensor, which was obtained for virtual Compton scattering $\gamma^{*}+e^{-} \rightarrow \gamma+e^{-}$is related to our case by proper analytic continuation; and indeed after analytic continuation we find agreement for the real part 4 contains our summary and the conclusions. The massdependent terms proportional to $m_{e}^{2}$ and $m_{e}^{4}$, expressed in the Cartesian basis, are given in Appendix A. The scalar loop integrals needed for the calculation are listed in Appendix B.

\section{The leptonic tensor for the radiative return}

Consider the $e^{+} e^{-}$annihilation process

$$
e^{+}\left(p_{1}\right)+e^{-}\left(p_{2}\right) \rightarrow \gamma^{*}(Q)+\gamma\left(k_{1}\right)
$$

where the virtual photon decays into a hadronic final state, $\gamma^{*}(Q) \rightarrow$ hadrons, and the real one is emitted from the initial state. The differential rate can be cast into the product of a leptonic and a hadronic tensor and the corresponding factorized phase space

$$
d \sigma=\frac{1}{2 s} L_{\mu \nu} H^{\mu \nu} d \Phi_{2}\left(p_{1}, p_{2} ; Q, k_{1}\right) d \Phi_{n}\left(Q ; q_{1}, \cdot, q_{n}\right) \frac{d Q^{2}}{2 \pi},
$$

\footnotetext{
1 We disagree, however, with eq.(30) of the translated version 23] which contains a missprint.
}

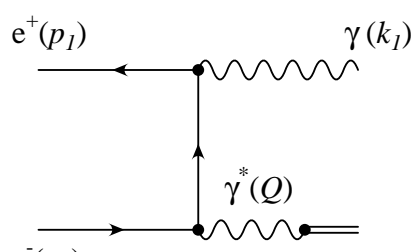

$\mathrm{e}^{-}\left(p_{2}\right)$

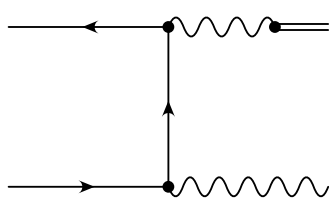

$\mathrm{M}_{0 \mathrm{~b}}$
Fig. 1. Initial-state radiation in the annihilation process $e^{+} e^{-} \rightarrow \gamma+$ hadrons at the Born level.

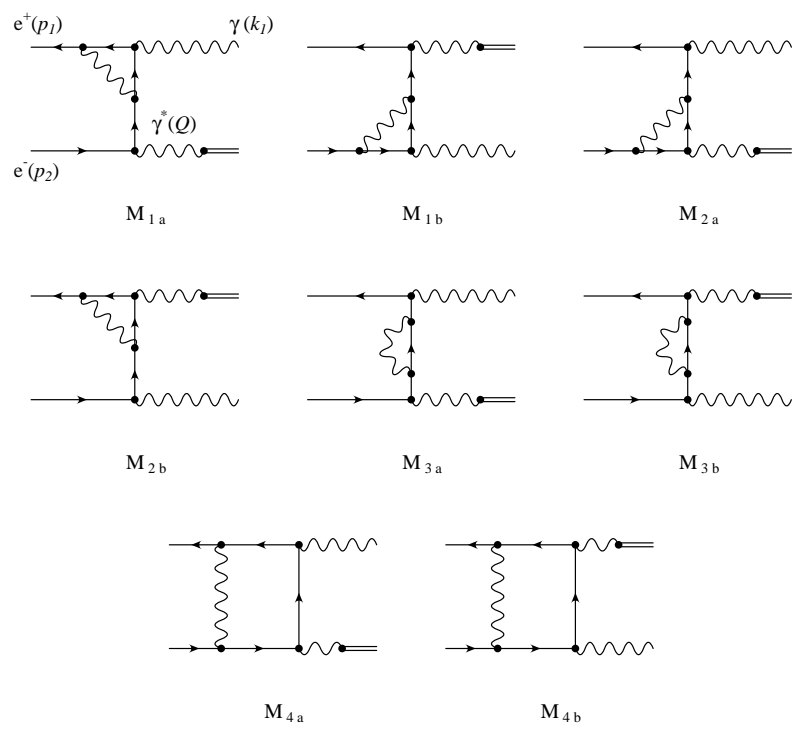

Fig. 2. One-loop corrections to initial-state radiation in $e^{+} e^{-}$ annihilation with the emission of a virtual photon.

where $d \Phi_{n}\left(Q ; q_{1}, \cdot, q_{n}\right)$ denotes the hadronic $n$-body phase space, including all the statistical factors coming from the hadronic final state.

For an arbitrary hadronic final state, the matrix element for the diagrams in Fig. 1 can be written

$$
M_{0}=M_{0}^{\mu} J_{\mu},
$$

where $J_{\mu}$ is the hadronic current and $M_{0}^{\mu}$ is the leptonic current in lowest order. Summing over the polarizations of the real photon, averaging over the polarizations of the initial $e^{+} e^{-}$state, and using current conservation, $Q_{\mu} J^{\mu}=0$, the leptonic tensor

$$
L_{0}^{\mu \nu}=\overline{M_{0}^{\mu} M_{0}^{\nu+}}
$$

can be written in the following form:

$$
\begin{aligned}
L_{0}^{\mu \nu} & =\frac{(4 \pi \alpha / s)^{2}}{q^{4}}\left[\left(\frac{2 m^{2} q^{2}\left(1-q^{2}\right)^{2}}{y_{1}^{2} y_{2}^{2}}-\frac{2 q^{2}+y_{1}^{2}+y_{2}^{2}}{y_{1} y_{2}}\right) g^{\mu \nu}\right. \\
& +\left(\frac{8 m^{2}}{y_{2}^{2}}-\frac{4 q^{2}}{y_{1} y_{2}}\right) \frac{p_{1}^{\mu} p_{1}^{\nu}}{s}+\left(\frac{8 m^{2}}{y_{1}^{2}}-\frac{4 q^{2}}{y_{1} y_{2}}\right) \frac{p_{2}^{\mu} p_{2}^{\nu}}{s} \\
& \left.-\left(\frac{8 m^{2}}{y_{1} y_{2}}\right) \frac{p_{1}^{\mu} p_{2}^{\nu}+p_{1}^{\nu} p_{2}^{\mu}}{s}\right],
\end{aligned}
$$


with

$$
y_{i}=\frac{2 k_{1} \cdot p_{i}}{s}, \quad m^{2}=\frac{m_{e}^{2}}{s}, \quad q^{2}=\frac{Q^{2}}{s} .
$$

It is symmetric under the exchange of the electron and the positron momenta. Expressing the bilinear products $y_{i}$ by the photon emission angle in the cms frame

$$
y_{1,2}=\frac{1-q^{2}}{2}(1 \mp \beta \cos \theta), \quad \beta=\sqrt{1-4 m^{2}},
$$

and rewriting the two-body phase space

$$
d \Phi_{2}\left(p_{1}, p_{2} ; Q, k_{1}\right)=\frac{1-q^{2}}{32 \pi^{2}} d \Omega
$$

it is evident that expression (14) contains several singularities: soft singularities for $q^{2} \stackrel{\rightarrow}{\rightarrow}$ and collinear singularities for $\cos \theta \rightarrow \pm 1$. The former are avoided by requiring a minimal photon energy. The latter are regulated by the electron mass.

The physics of the hadronic system, whose description is model-dependent, enters only through the hadronic tensor

$$
H^{\mu \nu}=J^{\mu} J^{\nu+},
$$

where the hadronic current has to be parametrized through form factors. For two charged pions in the final state, the current

$$
J_{2 \pi}^{\mu}=i e F_{2 \pi}\left(Q^{2}\right)\left(q_{\pi^{+}}-q_{\pi^{-}}\right)^{\mu},
$$

where $q_{\pi^{+}}$and $q_{\pi^{-}}$are the momenta of the $\pi^{+}$and $\pi^{-}$ respectively, is determined by only one function, the pion form factor $F_{2 \pi}$ [24. The hadronic current for four pions exhibits a more complicated structure and has been discussed in [5,25,26].

At NLO, the leptonic tensor receives contributions both from one-loop corrections arising from the insertion of virtual photon lines in the tree diagrams of Fig. 1 and from the emission of an extra real photon from the initial state. In this paper, we consider only the emission of soft photons. The implementation of these results in the program PHOKHARA and the discussion of their physical consequences will be considered in a separate work [27].

At NLO, the leptonic tensor has the following general form?:

$$
\begin{aligned}
L^{\mu \nu} & =\frac{(4 \pi \alpha / s)^{2}}{q^{4}}\left[a_{00} g^{\mu \nu}+a_{11} \frac{p_{1}^{\mu} p_{1}^{\nu}}{s}+a_{22} \frac{p_{2}^{\mu} p_{2}^{\nu}}{s}\right. \\
& \left.+a_{12} \frac{p_{1}^{\mu} p_{2}^{\nu}+p_{2}^{\mu} p_{1}^{\nu}}{s}+i \pi a_{-1} \frac{p_{1}^{\mu} p_{2}^{\nu}-p_{2}^{\mu} p_{1}^{\nu}}{s}\right] .
\end{aligned}
$$

Terms proportional to $Q^{\mu}$ are absent as a consequence of current conservation. The scalar coefficients $a_{i j}$ and $a_{-1}$ allow the following expansion

$$
a_{i j}=a_{i j}^{(0)}+\frac{\alpha}{\pi} a_{i j}^{(1)}, \quad a_{-1}=\frac{\alpha}{\pi} a_{-1}^{(1)} .
$$

${ }^{2}$ The present convention differs from the one in Ref. [18] by a factor $1 /\left(y_{1} y_{2}\right)$.
The LO coefficients $a_{i j}^{(0)}$ can be directly read from eq. (44)

$$
\begin{aligned}
& a_{00}^{(0)}=\frac{2 m^{2} q^{2}\left(1-q^{2}\right)^{2}}{y_{1}^{2} y_{2}^{2}}-\frac{2 q^{2}+y_{1}^{2}+y_{2}^{2}}{y_{1} y_{2}}, \\
& a_{11}^{(0)}=\frac{8 m^{2}}{y_{2}^{2}}-\frac{4 q^{2}}{y_{1} y_{2}}, \quad a_{22}^{(0)}=a_{11}^{(0)}\left(y_{1} \leftrightarrow y_{2}\right), \\
& a_{12}^{(0)}=-\frac{8 m^{2}}{y_{1} y_{2}} .
\end{aligned}
$$

The imaginary antisymmetric piece proportional to $a_{-1}$ appears for the first time at NLO. The leptonic tensor therefore remains fully symmetric only at LO.

As an alternative one can replace the Cartesian basis (eq. (9)) by a basis derived from the three circular polarization vectors of the virtual photon $\varepsilon_{L}$ and $\varepsilon_{ \pm}$, defined through

$$
\begin{aligned}
\varepsilon_{L}^{\mu}= & \frac{2\left(q^{\mu} q^{\nu}-g^{\mu \nu} q^{2}\right)}{\sqrt{s q^{2}}\left(1-q^{2}\right)} k_{1 \nu}, \\
\varepsilon_{1}^{\mu}= & \frac{2\left(q^{\mu} q^{\nu}-g^{\mu \nu} q^{2}\right)}{\sqrt{s} q^{2}\left(1-q^{2}\right)^{2} \beta \sin \theta}, \\
& \times\left(\left(y_{1}-q^{2} y_{2}\right) p_{1 \nu}-\left(y_{2}-q^{2} y_{1}\right) p_{2 \nu}\right), \\
\varepsilon_{2}^{\mu}= & \frac{2\left(q^{\mu} q^{\nu}-g^{\mu \nu} q^{2}\right)}{\sqrt{s^{3}} q^{2}\left(1-q^{2}\right) \beta \sin \theta} \epsilon_{\nu \eta \rho \sigma} k_{1}^{\eta} p_{1}^{\rho} p_{2}^{\sigma}, \\
\varepsilon_{ \pm}^{\mu}= & \frac{1}{\sqrt{2}}\left(\varepsilon_{1}^{\mu} \pm i \varepsilon_{2}^{\mu}\right),
\end{aligned}
$$

where $\epsilon_{\nu \eta \rho \sigma}$ is the four-dimensional totally antisymmetric Levi-Civita tensor, $\epsilon_{0123}=-1$. The leptonic tensor is thus given by

$$
L^{\mu \nu}=\frac{(4 \pi \alpha / s)^{2}}{q^{4}} \sum a_{i j} \varepsilon_{i}^{* \mu} \varepsilon_{j}^{\nu}, \quad i, j=L, \pm .
$$

Only four of the scalar coefficients are independent

$$
\begin{array}{ll}
a_{L-}=a_{L+}, & a_{-L}=a_{+L}=a_{L+}^{*}, \\
a_{--}=a_{++}, & a_{-+}=a_{+-} .
\end{array}
$$

The trace of the leptonic tensor

$$
L^{\mu \nu}\left(q_{\mu} q_{\nu}-g_{\mu \nu} q^{2}\right)=\frac{(4 \pi \alpha / s)^{2}}{q^{2}}\left(a_{L L}+2 a_{++}\right)
$$

is related to the cross section after angular averaging of the hadronic tensor.

The relations between the components in the Cartesian and the circular basis read as follows:

$$
\begin{aligned}
a_{L L} & =-a_{00}+\frac{1}{4 q^{2}\left(1-q^{2}\right)^{2}}\left[\left(y_{2}-q^{2} y_{1}\right)^{2} a_{11}\right. \\
& \left.+\left(y_{1}-q^{2} y_{2}\right)^{2} a_{22}+2\left(y_{1}-q^{2} y_{2}\right)\left(y_{2}-q^{2} y_{1}\right) a_{12}\right] \\
a_{L+} & =\frac{\beta \sin \theta}{4 \sqrt{2 q^{2}}\left(1-q^{2}\right)}\left[\left(y_{2}-q^{2} y_{1}\right) a_{11}-\left(y_{1}-q^{2} y_{2}\right) a_{22}\right. \\
& \left.+\left(1+q^{2}\right)\left(y_{1}-y_{2}\right) a_{12}-i \pi a_{-1}\right] \\
a_{+-} & =\frac{\beta^{2} \sin ^{2} \theta}{8}\left(a_{11}+a_{22}-2 a_{12}\right), \\
a_{++} & =a_{+-}-a_{00} .
\end{aligned}
$$


Conversely

$$
\begin{aligned}
a_{00} & =a_{+-}-a_{++} \\
a_{11} & =\frac{4}{\left(1-q^{2}\right)^{2}}\left[q^{2}\left(a_{L L}+a_{+-}-a_{++}\right),\right. \\
& +\frac{2 \sqrt{2 q^{2}}\left(y_{1}-q^{2} y_{2}\right)}{\left(1-q^{2}\right) \beta \sin \theta} \operatorname{Re}\left(a_{L+}\right) \\
& \left.+\frac{2\left(y_{1}-q^{2} y_{2}\right)^{2}}{\left(1-q^{2}\right)^{2} \beta^{2} \sin ^{2} \theta} a_{+-}\right], \\
a_{22} & =a_{11}\left(y_{1} \leftrightarrow y_{2}\right), \quad a_{12}=\frac{a_{11}+a_{22}}{2}-\frac{4 a_{+-}}{\beta^{2} \sin ^{2} \theta}, \\
a_{-1} & =-\frac{4 \sqrt{2 q^{2}}}{\pi\left(1-q^{2}\right) \beta \sin \theta} \operatorname{Im}\left(a_{L+}\right) .
\end{aligned}
$$

The scalar coefficients in the circular basis are given at LO by

$$
\begin{aligned}
& a_{L L}^{(0)}=\frac{2 q^{2} \beta^{2} \sin ^{2} \theta}{y_{1} y_{2}}, \\
& a_{L+}^{(0)}=\frac{\sqrt{q^{2}}\left(y_{1}-y_{2}\right) \beta \sin \theta}{\sqrt{2}\left(1-q^{2}\right) y_{1} y_{2}}\left(1+q^{2}-\frac{2 m^{2}\left(1-q^{2}\right)^{2}}{y_{1} y_{2}}\right) \\
& a_{+-}^{(0)}=\frac{\beta^{2} \sin ^{2} \theta}{y_{1} y_{2}}\left(-q^{2}+\frac{m^{2}\left(1-q^{2}\right)^{2}}{y_{1} y_{2}}\right) \\
& a_{++}^{(0)}=a_{+-}^{(0)}+\frac{2 q^{2}+y_{1}^{2}+y_{2}^{2}}{y_{1} y_{2}}-\frac{2 m^{2} q^{2}\left(1-q^{2}\right)^{2}}{y_{1}^{2} y_{2}^{2}}
\end{aligned}
$$

The one-loop matrix elements (Fig. 2) contribute to the leptonic tensor through their interference with the lowest order diagrams (Fig. 1). They contain ultraviolet (UV) and infrared (IR) divergences, which are regularized using dimensional regularization in $D=4-2 \varepsilon$ dimensions. The UV divergences are renormalized in the on-shell scheme. The IR divergences are cancelled by adding the contribution of an extra soft photon emitted from the initial state and integrated in the phase space up to an energy cutoff $E_{\gamma}<w \sqrt{s}$ far below $\sqrt{s}$. The result, which is finite, depends, however, on this soft photon cutoff. Only the contribution from hard photons with energy $E_{\gamma}>w \sqrt{s}$ would cancel this dependence.

The algebraic manipulations have been carried out with the help of the FeynCalc Mathematica package 28]. Using standard techniques [29], it automatically reduces the evaluation of the one-loop contribution to the calculation of a few scalar one-loop integrals and performs the Dirac algebra.

Since we consider the small angular region, mass terms proportional to $y_{i}^{-2}$ and even $y_{i}^{-3}$ arise. Terms proportional to $m^{2}$ and even $m^{4}$ must be kept, if they are multiplied by $y_{i}^{-2}$ and $y_{i}^{-3}$ respectively. In the expansion of the one-loop integrals, functions that depend on the ratio $\mathrm{m}^{2} / y_{i}$ cannot be expanded, in contrast to functions of $m^{2}$ or $y_{i}$ separately. To arrive at a systematic approach we therefore make the replacements $m^{2} \rightarrow \lambda m^{2}, y_{i} \rightarrow \lambda y_{i}$, perform the expansion for small $\lambda$ up to the appropriate order, and set $\lambda=1$ at the end.

\section{The NLO leptonic tensor}

Combining the one-loop and the soft contribution we now arrive at the leptonic tensor in NLO. It will be convenient to split the coefficients $a_{i j}^{(1)}$ into a part that contributes at large angles and a part proportional to $m^{2}$ and $m^{4}$, denoted by $a_{i j}^{(1,0)}$ and $a_{i j}^{(1, m)}$ respectively:

$$
\begin{aligned}
a_{i j}^{(1)} & =a_{i j}^{(0)}\left[-\log \left(4 w^{2}\right)\left[1+\log \left(m^{2}\right)\right]\right. \\
& \left.-\frac{3}{2} \log \left(\frac{m^{2}}{q^{2}}\right)-2+\frac{\pi^{2}}{3}\right]+a_{i j}^{(1,0)}+a_{i j}^{(1, m)} .
\end{aligned}
$$

The factor proportional to the LO coefficients $a_{i j}^{(0)}$ contains the usual soft and collinear logarithms. The expressions are particularly compact in the circular basis. For completeness we also repeat the results for $a_{i j}^{(1,0)}$, which can be found in [18], albeit in the Cartesian basis:

$$
\begin{aligned}
a_{L L}^{(1,0)} & =\frac{2 q^{2}}{\left(1-q^{2}\right)^{2}}\left\{-\frac{y_{1}}{1-y_{1}}-2 \log \left(q^{2}\right)+2 L\left(y_{1}\right)\right. \\
& \left.+\left[1-\frac{1-q^{2}}{1-y_{2}}-\frac{q^{2}}{\left(1-y_{2}\right)^{2}}\right] \log \left(\frac{y_{1}}{q^{2}}\right)+\left[y_{1} \leftrightarrow y_{2}\right]\right\},
\end{aligned}
$$

$$
\begin{aligned}
a_{L+}^{(1,0)} & =\frac{\beta \sin \theta}{4 \sqrt{2 q^{2}}\left(1-q^{2}\right)}\left\{\frac{y_{1}\left(1+q^{2}\right)^{2}}{\left(1-y_{1}\right)\left(1-y_{2}\right)}-\frac{\left(1-q^{2}\right)^{2}}{y_{2}}\right. \\
& -\frac{2 q^{2}\left(2+q^{2}\right)}{y_{2}} \log \left(q^{2}\right)+\left[2\left(1-q^{2}\right)\left(\frac{1}{y_{1}}-\frac{q^{2}}{y_{2}}\right)\right. \\
& \left.-\frac{2\left(1+q^{2}\right)}{1-y_{2}}-\frac{q^{2}\left(1+q^{2}\right)^{2}}{\left(1-y_{2}\right)^{2}}\right] \log \left(\frac{y_{1}}{q^{2}}\right) \\
& -2 q^{2}\left[\frac{1+2 q^{2}}{y_{1}}+\frac{y_{1}}{y_{2}^{2}}\right] L\left(y_{1}\right)-\frac{i \pi q^{2}}{y_{1} y_{2}}\left[\frac{2 \log \left(1-y_{1}\right)}{y_{1}}\right. \\
& \left.\left.+\frac{1-q^{2}}{1-y_{1}}+\frac{q^{2}}{\left(1-y_{1}\right)^{2}}\right]-\left[y_{1} \leftrightarrow y_{2}\right]\right\},
\end{aligned}
$$

$$
\begin{aligned}
a_{++}^{(1,0)} & =\frac{1}{2}\left\{\frac{1-y_{2}}{y_{1}}+\frac{1}{\left(1-q^{2}\right)^{2}}\left(\frac{1+q^{4}}{1-y_{1}}-2+2 \log \left(q^{2}\right)\right)+\right. \\
& +\frac{1}{1-q^{2}}\left[\frac{1+q^{4}}{1-q^{2}}\left(\frac{q^{2}}{\left(1-y_{2}\right)^{2}}-1\right)+\frac{3-q^{4}}{1-y_{2}}\right] \log \left(\frac{y_{1}}{q^{2}}\right) \\
& \left.+\frac{2}{1-q^{2}}\left[\frac{\left(1+q^{4}\right) y_{2}}{\left(1-q^{2}\right) y_{1}}+\frac{2}{y_{2}}\right] L\left(y_{1}\right)+\left[y_{1} \leftrightarrow y_{2}\right]\right\},
\end{aligned}
$$

\footnotetext{
3 The result for the imaginary part of $a_{L+}$ differs from $a_{-1}$ in the original version of 18 .
} 


$$
\begin{aligned}
a_{+-}^{(1,0)} & =\frac{q^{2}}{\left(1-q^{2}\right)^{2}}\left\{\frac{1+q^{4}}{2 q^{2}\left(1-y_{1}\right)}-\frac{1}{q^{2}}-\frac{y_{2}}{y_{1}} \log \left(q^{2}\right)\right. \\
& -\left[1+\frac{\left(1-q^{2}\right)^{2}}{y_{2}}-\frac{q^{2}\left(1-q^{2}\right)}{1-y_{2}}-\frac{1+q^{4}}{2\left(1-y_{2}\right)^{2}}\right] \\
& \left.\times \log \left(\frac{y_{1}}{q^{2}}\right)-\left[2+\frac{\left(1-q^{2}\right)^{2}}{y_{2}^{2}}\right] L\left(y_{1}\right)+\left[y_{1} \leftrightarrow y_{2}\right]\right\},
\end{aligned}
$$

with

$$
L\left(y_{i}\right)=L i_{2}\left(\frac{-y_{i}}{q^{2}}\right)-L i_{2}\left(1-\frac{1}{q^{2}}\right)+\log \left(q^{2}+y_{i}\right) \log \left(\frac{y_{i}}{q^{2}}\right),
$$

where $L i_{2}$ is the Spence or dilogarithm function. The result in the Cartesian basis has been given in Ref. [18]. The terms proportional to powers of $m^{2}$ are given by

$$
\begin{gathered}
a_{L L}^{(1, m)}=0 \\
a_{L+}^{(1, m)}=\frac{\sqrt{q^{2}} \beta \sin \theta}{4 \sqrt{2}}\left\{\frac { 4 m ^ { 2 } } { y _ { 1 } ^ { 2 } } \left[\log \left(q^{2}\right) \log \left(\frac{y_{1}^{4}}{m^{4} q^{2}}\right)\right.\right. \\
\left.+4 L i_{2}\left(1-q^{2}\right)+\frac{3}{2}\left(L i_{2}\left(1-\frac{y_{1}}{m^{2}}\right)-\frac{\pi^{2}}{6}\right)\right] \\
-n\left(y_{1}, \frac{2\left(1-3 q^{2}\right)}{q^{2}}\right)+\frac{2 m^{2} N\left(y_{1}\right)}{y_{1}\left(m^{2}\left(1-q^{2}\right)-y_{1}\right)} \\
\left.\quad-\left[y_{1} \leftrightarrow y_{2}\right]\right\}, \\
a_{++}^{(1, m)}=-\frac{m^{2} q^{2}}{y_{1}^{2}}\left[\log \left(q^{2}\right) \log \left(\frac{y_{1}^{4}}{m^{4} q^{2}}\right)+4 L i_{2}\left(1-q^{2}\right)\right. \\
\left.+L i_{2}\left(1-\frac{y_{1}}{m^{2}}\right)-\frac{\pi^{2}}{6}\right]-\frac{m^{2}\left(1-q^{2}\right)}{y_{1}^{2}}\left[1-\log \left(\frac{y_{1}}{m^{2}}\right)\right. \\
\left.+\frac{m^{2}}{y_{1}}\left(L i_{2}\left(1-\frac{y_{1}}{m^{2}}\right)-\frac{\pi^{2}}{6}\right)\right]+\frac{q^{2}}{2} n\left(y_{1}, \frac{1-3 q^{2}}{q^{2}}\right) \\
+\left[y_{1} \leftrightarrow y_{2}\right],
\end{gathered}
$$

and

$$
\begin{aligned}
a_{+-}^{(1, m)} & =\frac{\beta^{2} \sin ^{2} \theta}{8}\left\{\frac { 4 m ^ { 2 } } { y _ { 1 } ^ { 2 } } \left[\log \left(q^{2}\right) \log \left(\frac{y_{1}^{4}}{m^{4} q^{2}}\right)+4 L i_{2}\left(1-q^{2}\right)\right.\right. \\
& \left.+2\left(L i_{2}\left(1-\frac{y_{1}}{m^{2}}\right)-\frac{\pi^{2}}{6}\right)\right]-\frac{1-q^{2}}{q^{2}} n\left(y_{1}, \frac{3-7 q^{2}}{1-q^{2}}\right) \\
& +\frac{2 m^{2}\left(1-q^{2}\right)}{y_{1}\left(m^{2}\left(1-q^{2}\right)-y_{1}\right)}\left[\frac{1}{q^{2}} \log \left(\frac{y_{1}}{m^{2}}\right)+\frac{\log \left(q^{2}\right)}{1-q^{2}}\right. \\
& \left.+\left(\frac{3-q^{2}}{1-q^{2}}+\frac{m^{2}}{m^{2}\left(1-q^{2}\right)-y_{1}}\right) N\left(y_{1}\right)\right] \\
& \left.+\left[y_{1} \leftrightarrow y_{2}\right]\right\}
\end{aligned}
$$

The coefficient $a_{L+}$ is antisymmetric with respect to the exchange $\left[y_{1} \leftrightarrow y_{2}\right]$, while all the others are symmetric. Only $a_{L+}^{(1,0)}$ contributes to the imaginary part. Notice that the mass-suppressed terms are all real. The functions $n\left(y_{i}, z\right)$ and $N\left(y_{i}\right)$ are defined through

$$
\begin{aligned}
n\left(y_{i}, z\right) & =\frac{m^{2}}{y_{i}\left(m^{2}-y_{i}\right)}\left[1+z \log \left(\frac{y_{i}}{m^{2}}\right)\right] \\
& +\frac{m^{2}}{\left(m^{2}-y_{i}\right)^{2}} \log \left(\frac{y_{i}}{m^{2}}\right),
\end{aligned}
$$

$$
\begin{aligned}
N\left(y_{i}\right) & =\log \left(q^{2}\right) \log \left(\frac{y_{i}}{m^{2}}\right)+L i_{2}\left(1-q^{2}\right) \\
& +L i_{2}\left(1-\frac{y_{i}}{m^{2}}\right)-\frac{\pi^{2}}{6} .
\end{aligned}
$$

The apparent singularity of $n$ inside the limits of phase space is compensated by the zero in the numerator. For the numerical evaluation in the region $y_{i}$ close to $m^{2}$ we use

$$
\begin{aligned}
\left.n\left(y_{i}, z\right)\right|_{y_{i} \rightarrow m^{2}} & =\frac{1}{y_{i}}\left[1+z \log \left(\frac{y_{i}}{m^{2}}\right)\right] \\
& -\frac{1}{m^{2}} \sum_{n=0}\left(\frac{1}{n+2}+\frac{z}{n+1}\right)\left(1-\frac{y_{i}}{m^{2}}\right)^{n} .
\end{aligned}
$$

For the conversion from the circular to the Cartesian basis, and to ensure finite results in the limit $\sin \theta \rightarrow 0$, it is important that $a_{L+}$ and $a_{+-}$vanish at $\sim \sin \theta$ and $\sin ^{2} \theta$ respectively. This corresponds to the requirement that the factors in curly brackets do not diverge for small $\sin \theta$, i.e. in the limit $\left(m^{2}\left(1-q^{2}\right)-y_{i}\right) \rightarrow 0$. This is guaranteed by the behaviour of $N\left(y_{i}\right)$ for $y_{i} \rightarrow m^{2}\left(1-q^{2}\right)$ :

$$
\left.\frac{m^{2} N\left(y_{i}\right)}{m^{2}\left(1-q^{2}\right)-y_{i}}\right|_{y_{i} \rightarrow m^{2}\left(1-q^{2}\right)}=-\frac{\log \left(1-q^{2}\right)}{q^{2}}-\frac{\log \left(q^{2}\right)}{1-q^{2}},
$$

The results for $a_{i j}^{(1, m)}$ in the Cartesian basis are listed in Appendix A.

We note that the imaginary part of $L_{\mu \nu}$, which is present in the coefficients $a_{L+}$ or $a_{-1}$ only, is of interest for those cases where the hadronic current receives contributions from different amplitudes with non-trivial relative phases. This is possible, e.g. for final states with three or more mesons or for $p \bar{p}$ production.

\section{Tests of the result}

After summation over the polarizations of the virtual photon the differential rate is given by the properly contracted 
leptonic tensor:

$$
\begin{aligned}
& \frac{q^{2}}{(4 \pi \alpha / s)^{2}} L^{\mu \nu}\left(q_{\mu} q_{\nu}-g_{\mu \nu} q^{2}\right)=a_{L L}+2 a_{++} \\
& =-3 a_{00}+\left(\frac{1}{4 q^{2}}\left(1-y_{1}\right)^{2}-m^{2}\right) a_{11} \\
& +\left(\frac{1}{4 q^{2}}\left(1-y_{2}\right)^{2}-m^{2}\right) a_{22} \\
& +\left(\frac{1}{2 q^{2}}\left(1-y_{1}\right)\left(1-y_{2}\right)-\left(1-2 m^{2}\right)\right) a_{12} \\
& =L_{0}\left\{1+\frac{\alpha}{\pi}\left[-\log \left(4 w^{2}\right)\left[1+\log \left(m^{2}\right)\right]\right.\right. \\
& \left.\left.-\frac{3}{2} \log \left(\frac{m^{2}}{q^{2}}\right)-2+\frac{\pi^{2}}{3}\right]\right\}+\frac{\alpha}{\pi}\left\{4 \left(\frac{1+\left(1-y_{2}\right)^{2}}{2 y_{1} y_{2}} L\left(y_{1}\right)\right.\right. \\
& +\frac{1-2 q^{2}}{2\left(1-q^{2}\right)^{2}} \log \left(q^{2}\right)+\left[1-\frac{y_{1}-2 y_{2}}{2\left(1-y_{2}\right)}-\frac{y_{1} y_{2}}{4\left(1-y_{2}\right)^{2}}\right] \\
& \times \log \left(\frac{y_{1}}{q^{2}}\right)-\frac{1}{2\left(1-q^{2}\right)}+\frac{1}{4\left(1-y_{1}\right)}+\frac{1-y_{2}}{4 y_{1}} \\
& \left.\left.+\left[y_{1} \leftrightarrow y_{2}\right]\right)-2 a_{++}^{(1, m)}\right\},
\end{aligned}
$$

where

$$
\begin{aligned}
L_{0} & =\frac{q^{2}}{(4 \pi \alpha / s)^{2}} L_{0}^{\mu \nu}\left(q_{\mu} q_{\nu}-q^{2} g_{\mu \nu}\right)= \\
& -\frac{2}{y_{1} y_{2}}\left[2 q^{2}+y_{1}^{2}+y_{2}^{2}-\frac{2 m^{2} q^{2}\left(1-q^{2}\right)^{2}}{y_{1} y_{2}}\right]
\end{aligned}
$$

$L_{0}^{\mu \nu}$ being the leptonic tensor at LO. In $L_{0}$ only the relevant terms in the limit $m^{2} \rightarrow 0$ have been kept. Large angle terms and mass corrections are in agreement with Berends et al. [20,21]. After proper analytic continuation and using the substitutions

$$
t \rightarrow-y_{1}+i \eta, \quad s \rightarrow-y_{2}+i \eta, \quad u \rightarrow s+i \eta
$$

the leptonic tensor in eq. (9) is closely related to the tensor $T_{\mu \nu}$, which describes "virtual Compton scattering" and has been calculated by Kuraev et al. 22]. After performing this analytical continuation, the results are in agreement (However, the result for $T_{12}$ printed in [23] contains a typo and would give rise to a discrepancy.).

\section{Conclusions}

Compact analytical formulae have been obtained for the virtual corrections to the process $e^{+} e^{-} \rightarrow \gamma \gamma^{*}$, which are valid for photon emission under both large and small angles. After proper analytic continuation the results are in agreement with those obtained in 22 for the reaction $\gamma^{*}+e^{-} \rightarrow \gamma+e^{-}$. In polarization averaged form they are in agreement with those for $e^{+} e^{-} \rightarrow \gamma Z$ from [20,21].

\footnotetext{
${ }^{4}$ We thank N.P. Merenkov for drawing our attention to this reference.
}

\section{Acknowledgements}

We would like to thank G. Cataldi, A. Denig, S. Di Falco, W. Kluge, S. Müller, G. Venanzoni and B. Valeriani for reminding us constantly of the importance of this work for the experimental analysis and H. Czyż and N.P. Merenkov for very useful discussions. Work supported in part by BMBF under grant number 05HT9VKB0 and E.U. EURODA $\Phi$ NE network TMR project FMRX-CT98-0169.

\section{A The leptonic tensor in the Cartesian basis}

For the convenience of the reader we list the mass-suppressed terms $a_{i j}^{(1, m)}$ also in the Cartesian basis. The large-angle contributions have been given in 18 . The component proportional to $g_{\mu \nu}$ reads

$$
a_{00}^{(1, m)}=-a_{++}^{(1, m)}
$$

see eq. (25). The coefficient $a_{+-}$does not contribute at this order to $a_{00}$. The remaining components are given by

$$
\begin{aligned}
& a_{11}^{(1, m)}=\frac{q^{2}}{1-q^{2}}\left\{\frac { 4 m ^ { 2 } } { y _ { 1 } ^ { 2 } } \left[1-\log \left(\frac{y_{1}}{m^{2}}\right)\right.\right. \\
& \left.+\frac{m^{2}}{y_{1}}\left(L i_{2}\left(1-\frac{y_{1}}{m^{2}}\right)-\frac{\pi^{2}}{6}\right)\right]-n\left(y_{1}, 1\right) \\
& +\frac{2 m^{2} q^{2}}{y_{1}\left(m^{2}\left(1-q^{2}\right)-y_{1}\right)}\left[\frac{1}{q^{2}} \log \left(\frac{y_{1}}{m^{2}}\right)+\frac{\log \left(q^{2}\right)}{1-q^{2}}\right. \\
& \left.\left.+\left(1+\frac{m^{2}}{m^{2}\left(1-q^{2}\right)-y_{1}}\right) N\left(y_{1}\right)\right]\right\}+ \\
& +\frac{1}{1-q^{2}}\left\{\frac { 4 m ^ { 2 } ( 1 - q ^ { 2 } ) } { y _ { 2 } ^ { 2 } } \left[\log \left(q^{2}\right) \log \left(\frac{y_{2}^{4}}{m^{4} q^{2}}\right)\right.\right. \\
& \left.+4 L i_{2}\left(1-q^{2}\right)+2\left(L i_{2}\left(1-\frac{y_{2}}{m^{2}}\right)-\frac{\pi^{2}}{6}\right)\right] \\
& +\frac{4 m^{2} q^{2}}{y_{2}^{2}}\left[1-\log \left(\frac{y_{2}}{m^{2}}\right)+\left(1+\frac{m^{2}}{y_{2}}\right)\left(L i_{2}\left(1-\frac{y_{2}}{m^{2}}\right)\right.\right. \\
& \left.\left.-\frac{\pi^{2}}{6}\right)\right]-\frac{1-2 q^{4}}{q^{2}} n\left(y_{2}, \frac{3-8 q^{2}+6 q^{4}}{1-2 q^{4}}\right) \\
& +\frac{2 m^{2}}{y_{2}\left(m^{2}\left(1-q^{2}\right)-y_{2}\right)}\left[\frac{1}{q^{2}} \log \left(\frac{y_{2}}{m^{2}}\right)+\frac{\log \left(q^{2}\right)}{1-q^{2}}\right. \\
& \left.\left.+\left(3+\frac{m^{2}}{m^{2}\left(1-q^{2}\right)-y_{2}}\right) N\left(y_{2}\right)\right]\right\} \text {, }
\end{aligned}
$$

$$
a_{22}^{(1, m)}=a_{11}^{(1, m)}\left(y_{1} \leftrightarrow y_{2}\right)
$$




$$
\begin{aligned}
a_{12}^{(1, m)} & =\frac{q^{2}}{1-q^{2}}\left\{\frac { 4 m ^ { 2 } } { y _ { 1 } ^ { 2 } } \left[1-\log \left(\frac{y_{1}}{m^{2}}\right)\right.\right. \\
& \left.+\left(\frac{1}{2}+\frac{m^{2}}{y_{1}}\right)\left(L i_{2}\left(1-\frac{y_{1}}{m^{2}}\right)-\frac{\pi^{2}}{6}\right)\right] \\
& -\frac{1-q^{2}}{q^{2}} n\left(y_{1}, \frac{1}{1-q^{2}}\right)+\frac{2 m^{2}}{y_{1}\left(m^{2}\left(1-q^{2}\right)-y_{1}\right)} \\
& \times\left[\frac{1}{q^{2}} \log \left(\frac{y_{1}}{m^{2}}\right)+\frac{\log \left(q^{2}\right)}{1-q^{2}}\right. \\
& \left.\left.+\left(2+\frac{m^{2}}{m^{2}\left(1-q^{2}\right)-y_{1}}\right) N\left(y_{1}\right)\right]\right\}+\left[y_{1} \leftrightarrow y_{2}\right]
\end{aligned}
$$

and

$$
a_{-1}^{(1, m)}=0
$$

\section{B Scalar one-loop integrals}

A few two-, three-, and four-point scalar one-loop integrals enter our calculation. Expression for the two-point scalar integrals are simple and well known. The notation from [18], where the corresponding results valid for large photon angles $\left(m^{2} \ll 1, q^{2}, y_{i}\right)$ can be found, is used in the following. The general three-point scalar one-loop integral is defined by

$$
\begin{aligned}
& C_{0}\left(p_{a}^{2},\left(p_{a}-p_{b}\right)^{2}, p_{b}^{2}, m_{1}^{2}, m_{2}^{2}, m_{3}^{2}\right)=-i 16 \pi^{2} \mu^{4-D} \\
& \times \int \frac{d^{D} k}{(2 \pi)^{D}} \frac{1}{\left[k^{2}-m_{1}^{2}\right]\left[\left(k-p_{a}\right)^{2}-m_{2}^{2}\right]\left[\left(k-p_{b}\right)^{2}-m_{3}^{2}\right]} .
\end{aligned}
$$

Four different three-point scalar one-loop integrals are needed

$$
\begin{aligned}
& C 01=C_{0}\left(\left(p_{i}-k_{1}\right)^{2}, 0, m_{e}^{2}, 0, m_{e}^{2}, m_{e}^{2}\right), \\
& C 02=C_{0}\left(m_{e}^{2}, s, m_{e}^{2}, 0, m_{e}^{2}, m_{e}^{2}\right), \\
& C 03=C_{0}\left(\left(p_{i}-k_{1}\right)^{2}, Q^{2}, m_{e}^{2}, 0, m_{e}^{2}, m_{e}^{2}\right), \\
& C 04=C_{0}\left(Q^{2}, s, 0, m_{e}^{2}, m_{e}^{2}, m_{e}^{2}\right),
\end{aligned}
$$

$i=1,2$, and one scalar box

$D 0=-i 16 \pi^{2} \mu^{4-D} \int \frac{d^{D} k}{(2 \pi)^{D}}$

$$
\times \frac{1}{k^{2}\left[\left(k+p_{i}\right)^{2}-m_{e}^{2}\right]\left[\left(k+p_{i}-k_{1}\right)^{2}-m_{e}^{2}\right]\left[\left(k-p_{j}\right)^{2}-m_{e}^{2}\right]}
$$

with $j \neq i$.

The following simple expressions are used, from where the limits $\left(m^{2} \ll 1, q^{2}, y_{i}\right)$ or $\left(m^{2} \ll 1, q^{2}\right.$ but $\left.m^{2} \sim y_{i}\right)$ are obtained:

$$
\begin{aligned}
& C 01=\frac{s^{-1}}{y_{i}}\left[L i_{2}\left(1-\frac{y_{i}}{m^{2}}\right)-\frac{\pi^{2}}{6}\right] \\
& \begin{aligned}
C 02 & =\frac{s^{-1}}{\beta}\left[\left(\Delta-2 \log (\beta)-\frac{\log (c)}{2}\right) \log (c)\right. \\
- & \left.2 L i_{2}(c)-\frac{2 \pi^{2}}{3}+i \pi(\Delta-2 \log (\beta))\right] \\
C 04 & =\frac{s^{-1}}{1-q^{2}}\left[\frac{\log (c)}{2}-\frac{\log \left(c_{q}\right)}{2}+i \pi \log \left(\frac{c}{c_{q}}\right)\right] \\
D 0 & =\frac{s^{-2}}{\beta y_{i}}\left[-\left(\Delta+2 \log \left(\frac{m}{y_{i}}\right)\right) \log \left(c_{q}\right)+\log ^{2}(c)\right. \\
+ & 2 L i_{2}\left(1-\frac{c_{q}}{c}\right)+2 L i_{2}\left(1-c c_{q}\right)-L i_{2}\left(1-c_{q}^{2}\right)-\pi^{2} \\
- & \left.i \pi\left(\Delta+2 \log \left(\frac{m}{c y_{i}}\right)\right)\right]
\end{aligned}
\end{aligned}
$$

with

$$
\Delta=\frac{(4 \pi)^{\varepsilon}}{\varepsilon \Gamma(1-\varepsilon)}\left(\frac{\mu^{2}}{s}\right)^{\varepsilon}
$$

and

$$
\begin{aligned}
\beta & =\sqrt{1-4 m^{2}}, \quad c=\frac{1-\beta}{1+\beta}, \\
\beta_{q} & =\sqrt{1-\frac{4 m^{2}}{q^{2}}}, \quad c_{q}=\frac{1-\beta_{q}}{1+\beta_{q}} .
\end{aligned}
$$

Our expression for the $\mathrm{C} 03$ function is rather cumbersome:

$$
\begin{aligned}
C 03 & =\frac{s^{-1}}{q^{2}\left(z_{1}-z_{2}\right)}\left[\log \left(\frac{y_{i}}{q^{2}}\right) \log \left(\frac{\left(1-z_{1}\right) z_{2}}{\left(1-z_{2}\right) z_{1}}\right)\right. \\
& +\left\{L i_{2}\left(\frac{1}{z_{1}}\right)-\log \left(\left(z_{3}-z_{1}\right)\left(z_{4}-z_{1}\right)\right) \log \left(1-\frac{1}{z_{1}}\right)\right. \\
& +L i_{2}\left(\frac{1-z_{1}}{z_{3}-z_{1}}\right)-L i_{2}\left(\frac{-z_{1}}{z_{3}-z_{1}}\right) \\
& \left.\left.+L i_{2}\left(\frac{1-z_{1}}{z_{4}-z_{1}}\right)-L i_{2}\left(\frac{-z_{1}}{z_{4}-z_{1}}\right)-\left[z_{1} \leftrightarrow z_{2}\right]\right\}\right],
\end{aligned}
$$

where

$$
\begin{aligned}
& z_{1,2}=\frac{q^{2}+y_{i}}{2 q^{2}}\left(1 \pm \sqrt{1-\frac{4\left(m^{2}-i \eta\right) q^{2}}{\left(q^{2}+y_{i}\right)^{2}}}\right) \\
& z_{3,4}=\frac{1}{2}\left(1 \pm \sqrt{1-\frac{4\left(m^{2}-i \eta\right)}{q^{2}}}\right)
\end{aligned}
$$

being

$$
\operatorname{Im}(C 03)=\frac{\pi}{q^{2}\left(z_{1}-z_{2}\right)} \log \left(\frac{\left(z_{3}-z_{1}\right)\left(z_{4}-z_{2}\right)}{\left(z_{4}-z_{1}\right)\left(z_{3}-z_{2}\right)}\right)
$$

its imaginary part. As for the other scalar one-loop functions, this expression is expanded up to terms of order $m^{2}$ and $m^{4}$. 


\section{References}

1. [LEP Collaborations], hep-ex/0103048.

2. J. H. Kühn and M. Steinhauser, Nucl. Phys. B 619 (2001) 588 hep-ph/0109084.

3. S. Binner, J. H. Kühn and K. Melnikov, Phys. Lett. B459 (1999) 279 hep-ph/9902399.

4. K. Melnikov, F. Nguyen, B. Valeriani and G. Venanzoni, Phys. Lett. B477 (2000) 114 hep-ph/0001064.

5. H. Czyż and J. H. Kühn, Eur. Phys. J. C 18 (2001) 497 hep-ph/0008262.

6. S. Spagnolo, Eur. Phys. J. C 6 (1999) 637.

7. V. A. Khoze, M. I. Konchatnij, N. P. Merenkov, G. Pancheri, L. Trentadue and O. N. Shekhovzova, Eur. Phys. J. C 18 (2001) 481 hep-ph/0003313.

8. A. Höfer, J. Gluza and F. Jegerlehner, hep-ph/0107154.

9. V. A. Khoze, M. I. Konchatnij, N. P. Merenkov, G. Pancheri, L. Trentadue and O. N. Shekhovtzova, hep$\mathrm{ph} / 0202021$.

10. A. Aloisio et al. [KLOE Collaboration], hep-ex/0107023.

11. A. Denig et al. [KLOE Collaboration], eConf C010430 (2001) T07 hep-ex/0106100.

12. M. Adinolfi et al. [KLOE Collaboration], hep-ex/0006036.

13. E. P. Solodov [BABAR collaboration], eConf C010430 (2001) T03 hep-ex/0107027.

14. M. Caffo, H. Czyż and E. Remiddi, Nuovo Cim. A 110 (1997) 515 hep-ph/9704443; Phys. Lett. B327 (1994) 369.

15. G. Rodrigo, H. Czyż, J. H. Kühn and M. Szopa, hep$\mathrm{ph} / 0112184$

16. G. 't Hooft and M. J. Veltman, Nucl. Phys. B 153 (1979) 365 .

17. W. Beenakker and A. Denner, Nucl. Phys. B 338 (1990) 349.

18. G. Rodrigo, A. Gehrmann-De Ridder, M. Guilleaume and J. H. Kühn, Eur. Phys. J. C 22 (2001) 81 hep$\mathrm{ph} / 0106132$.

19. G. Rodrigo Acta Phys. Polon. B 32 (2001) 3833 hep$\mathrm{ph} / 0111151$.

20. F. A. Berends, G. J. Burgers and W. L. van Neerven, Phys. Lett. B177 (1986) 191.

21. F. A. Berends, W. L. van Neerven and G. J. Burgers, Nucl. Phys. B297 (1988) 429; Erratum Nucl. Phys. B304 (1988) 921.

22. E. A. Kuraev, N. P. Merenkov and V. S. Fadin, Yad. Fiz. 45 (1987) 782.

23. E. A. Kuraev, N. P. Merenkov and V. S. Fadin, Sov. J. Nucl. Phys. 45 (1987) 486.

24. J. H. Kühn and A. Santamaria, Z. Phys. C 48 (1990) 445.

25. R. Decker, M. Finkemeier, P. Heiliger and H. H. Jonsson, Z. Phys. C 70 (1996) 247 hep-ph/9410260.

26. G. Ecker and R. Unterdorfer, hep-ph/0203075.

27. G. Rodrigo, H. Czyż and J. H. Kühn, in preparation.

28. R. Mertig, M. Böhm and A. Denner, Comput. Phys. Commun. 64 (1991) 345.

29. G. Passarino and M. Veltman, Nucl. Phys. B 160 (1979) 151. 